\title{
A WAVELENGTH-PRESERVING ALL-OPTICAL 3R REGENERATOR
}

A novel all-optical $3 R$ regenerator operated in the optically clocked scheme $(O C-3 R)$ is presented. The regenerator basically comprises two semiconductor components: an ultra-long semiconductor optical amplifier (UL-SOA) for effective use of fast nonlinearities and a self-pulsating PhaseCOMB-laser for all-optical clock recovery. The dynamic behaviour of the key devices is investigated theoretically and experimentally. An excellent regenerative performance of the assembled OC-3R is demonstrated by eye analysis and by bit error rate (BER) measurements at $40 \mathrm{Gbit} / \mathrm{s}$.

\section{Introduction}

In future high-speed global optical networks the data signals will be transparently switched through the nodes without additional opto-electronic conversion. In this case all-optical $3 \mathrm{R}$ regeneration (re-amplification, re-timing, re-shaping) is a key function in order to make networks resistant to distortions derived from optical cross-connects and transmission links [1]. A scheme of a $3 \mathrm{R}$ regenerator with its three functional elements is depicted in Fig. 1. First, the power level of the degraded signal is increased in the amplification stage. The re-timing function is performed by the clock signal, which is recovered from the pseudo random bit sequence (PRBS) data signal. Finally, a decision stage with a threshold function suppresses accumulated noise and equalises amplitude fluctuations. A completely restored signal is obtained at the regenerator output.

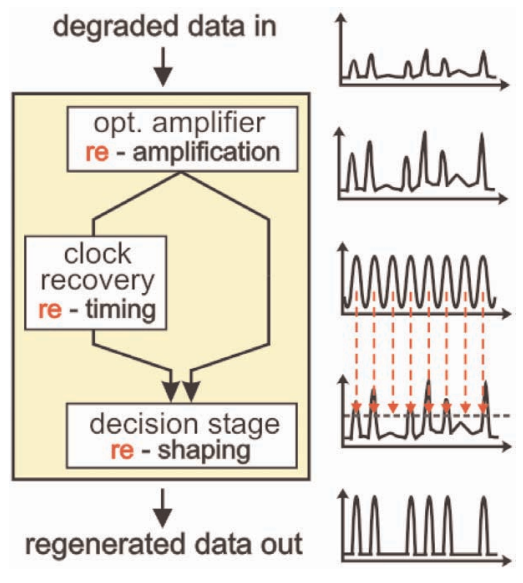

Fig. 1 Principle scheme of a $3 R$ regenerator [1]

Several regenerator architectures have been demonstrated in different experiments [2,3]. However, most solutions apply a large number of optical components and require high power levels inside the regenerator. In this paper we present a novel regenerator scheme that is based on only two compact semiconductor devices: a $4 \mathrm{~mm}$ ultra-long semiconductor optical amplifier (UL-SOA) for effective exploitation of fast non-linearities and a self-pulsating DFB laser (PhaseCOMB) for all-optical clock recovery. An optically clocked technique is applied to stabilise the gain dynamic in the UL-SOA and to perform the regeneration function on the data signal [4]. The application of compact semiconductor devices results in low power consumption and integration possibility on a planar lightwave circuit platform. We investigate theoretically and experimentally the characteristics of the key components and demonstrate the performance of the assembled optically clocked $3 \mathrm{R}$ regenerator at $40 \mathrm{Gbit} / \mathrm{s}$.

\section{Optically clocked all-optical $3 R$ regenerator}

The experimental set-up of the optically clocked regenerator (OC-3R) is shown in Fig. 2. An ideal 40 Gbit/s RZ PRBS signal at $1550 \mathrm{~nm}$ was first degraded in an optical noise unit in order to simulate the distortions derived from a transmission link. At the regenerator input the data signal was amplified by a linear semiconductor optical amplifier (LOA). A split-off part of the signal was injected into the self-pulsating PhaseCOMB laser for all-optical clock recovery. The PhaseCOMB laser synchronised to the injected data signal and emitted a $40 \mathrm{GHz}$ stable sinusoidal pulse stream at $1562 \mathrm{~nm}$. The second part of the data signal was delayed by half a bit relative to the clock pulses. Both signals were simultaneously

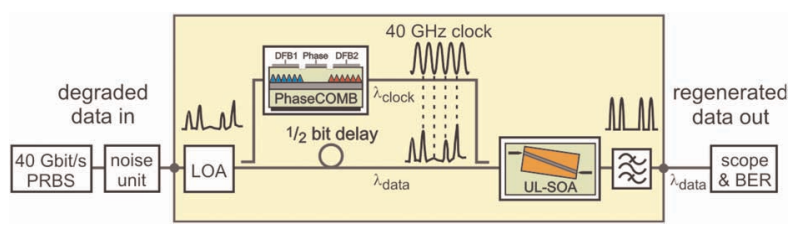

Fig. 2 Set-up of the all-optical OC-3R regenerator

\footnotetext{
* J. Slovak, C. Bornholdt, B. Sartorius

Fraunhofer Institute for Telecommunications, Heinrich-Hertz-Institut, Einsteinufer 37, 10587 Berlin, Germany
} 
launched into the ultra-long SOA. The clock signal was blocked at the regenerator output while the data signal was transmitted on its original wavelength. The quality of the data signal was analysed by jitter analysis using an electrical sampling oscilloscope and by BER measurements. In the following, the key devices of the OC-3R are characterised in more detail.

\section{Ultra-long semiconductor optical amplifier (UL-SOA)}

An ultra-long semiconductor optical amplifier with a length of $4 \mathrm{~mm}$ has been developed and manufactured (Fig 3). It is based on a BH structure with a $1.5 \mu \mathrm{m} \mathrm{InGaAsP} \mathrm{strained} \mathrm{bulk} \mathrm{active} \mathrm{layer}$ for polarisation insensitive operation. Tilted facets are applied additionally to the antireflection coating in order to minimise unwanted reflections. The UL-SOA chip is packaged into a Peltier-cooled module.
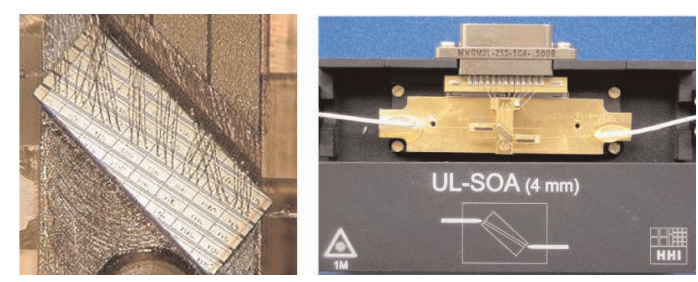

Fig. 3 The chip of the $4 \mathrm{~mm}$ UL-SOA (left-hand side) and the device packaged into a module (right-hand side)

\subsection{Numerical results on UL-SOA gain dynamic at $40 \mathrm{Gbit} / \mathrm{s}$}

The dynamic behaviour of an SOA was simulated using the VPI Transmission Maker program. First a single ideal $40 \mathrm{Gbit} / \mathrm{s}$ RZ data signal (Fig. 4a) was injected into the SOA with a length of $4 \mathrm{~mm}$. Strong signal degradation caused by patterning effects can be observed (Fig. 4b). Due to the relatively slow inter-band effects [5] the data pulses are amplified differently depending on the previous data sequence passing through the SOA. The first " 1 " bit after a long "zeros" sequence is amplified more then a " 1 " within a bit sequence of "ones". Next, the optically clocked principle was applied. In this case, an additional $40 \mathrm{GHz}$ sinusoidal signal is launched into the SOA simultaneously with the data signal.
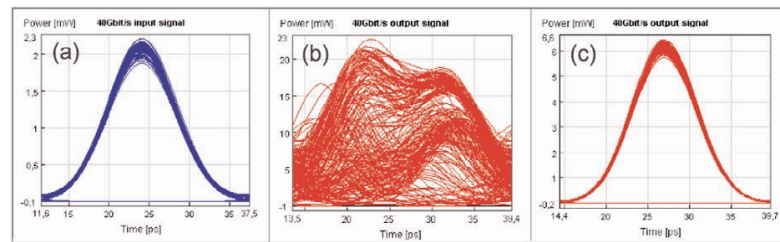

Fig. 4 Eye diagrams of the ideal $40 \mathrm{Gbit} / \mathrm{s} R Z$ signal at the SOA input (a), and of the output data signal without additional SOA stabilisation (b) and by applying the OC-technique (c)
The clock pulses are delayed by half a bit relative to the data pulses, which is the crucial condition of this principle. The corresponding data signal at the SOA output is shown in Fig. 4c. The slow gain dynamic is suppressed by the strong clock signal. Thus the high signal quality is preserved.

The behaviour of the optically clocked SOA was simulated also for degraded $40 \mathrm{Gbit} / \mathrm{s}$ PRBS data signals (Fig. 5a). The length of the SOA varied from $0.5 \mathrm{~mm}$ to $8 \mathrm{~mm}$ and the corresponding quality of the data signal at the SOA output was analysed (Fig. 5b-f). A signal improvement mainly for longer devices can be observed. The interaction between data and clock leads to the regenerative function due to fast intra-band effects in the semiconductor medium. However, comparing the simulation results for the $4 \mathrm{~mm}$ and the $8 \mathrm{~mm}$ SOA reveals only a relatively small improvement of the quality. Note, for practical use the SOA should be as short as possible. Thus a $4 \mathrm{~mm}$ UL-SOA has been manufactured for experimental analysis.

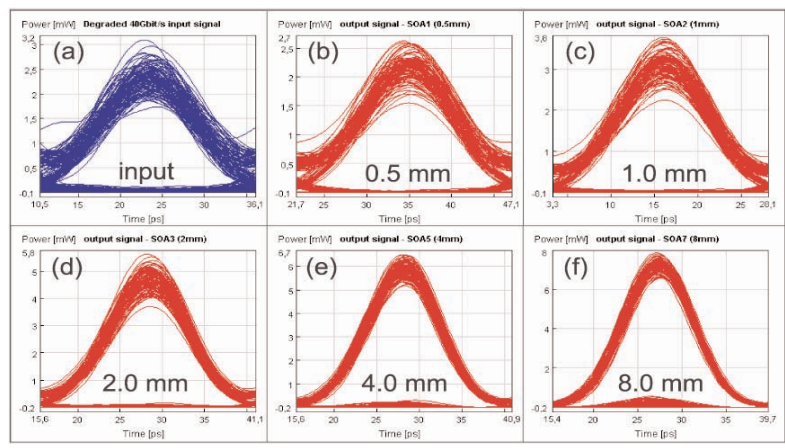

Fig. 5 Degraded data signal at the SOA input

(a) and the corresponding output signals for different SOA lengths (b-f) by applying the OC-principle

\subsection{Experimental investigation of the UL-SOA performance at $40 \mathrm{Gbit} / \mathrm{s}$}

The dynamic behaviour of the $4 \mathrm{~mm}$ UL-SOA at $40 \mathrm{Gbit} / \mathrm{s}$ was investigated experimentally. We analysed the results using an

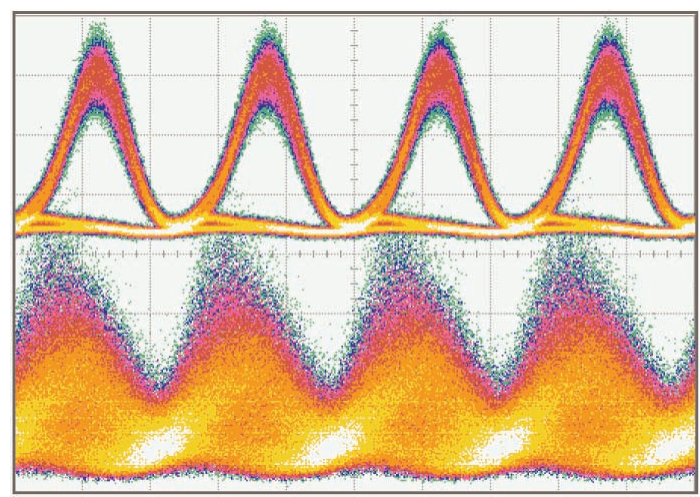

Fig. 6 Eye diagram of 40Gbit/s PRBS RZ signal (upper trace) and the data signal at the UL-SOA output (lower trace) 
electrical sampling oscilloscope with precision time base. First, a high-quality $40 \mathrm{Gbit} / \mathrm{s} \mathrm{RZ}$ data signal at $1550 \mathrm{~nm}$ (Fig. 6 upper trace) was launched directly into the UL-SOA. As expected from the simulation results, strong signal degradation due to patterning effects occurs (Fig. 6 lower trace).

The optically clocked technique was applied in order to stabilise the dynamic of the UL-SOA. An additional $40 \mathrm{GHz}$ sinusoidal signal at $1560 \mathrm{~nm}$ was injected into the device. The clock signal was generated using an external modulator electrically driven by a $40 \mathrm{GHz}$ synthesizer. In this case, no signal degradation is observed at the output of the semiconductor device (Fig. 7 lower trace). The carrier density in the ultra-long SOA is periodically modulated by the clock signal and not by the pattern envelope of the PRBS signal. This validates the analysis performed by simulations.

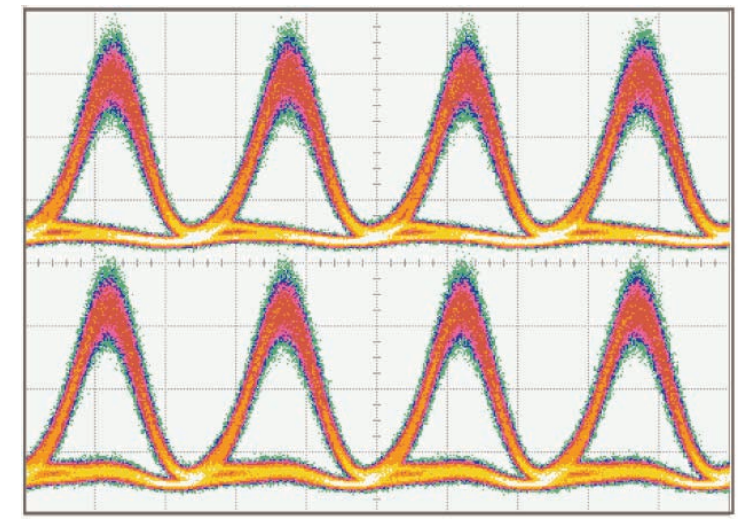

Fig. $740 \mathrm{Gbit/s}$ PRBS RZ signal at the UL-SOA input (upper trace) and the output data signal (lower trace). The UL-SOA is stabilised by an additional $40 \mathrm{GHz}$ clock signal

\subsection{Jitter reduction function of the optically clocked UL-SOA}

It was shown that the quality of the ideal data signal remains preserved at the output of the UL-SOA applying the optically

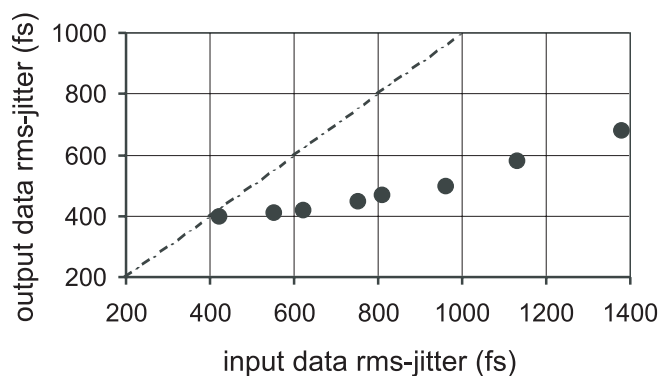

Fig. 8 Rms-jitter of the PRBS RZ signal measured by a sampling oscilloscope at the input of the optically clocked UL-SOA and the corresponding time jitter of the output data signal clocked technique. Next, we continuously degraded the data signal by additional time jitter and measured the corresponding jitter of the output signal. The power levels of the $40 \mathrm{Gbit} / \mathrm{s}$ data signal and $40 \mathrm{GHz}$ clock signal at the UL-SOA facet were $-2 \mathrm{dBm}$ and $+4 \mathrm{dBm}$ respectively. The results are summarised in Fig. 8. The rms-jitter of the input signal varies in a wide range from $400 \mathrm{fs}$ to $1400 \mathrm{fs}$. A strong jitter reduction function of the optically clocked ULSOA can be observed. The rms-jitter of the output signal increases only slightly and remains below $700 \mathrm{fs}$. The jitter improvement is related to the clock signal with its high time stability. The periodic gain modulation of the ultra-long SOA by the stable sinusoidal signal effectively shapes the data pulses and pushes them towards the correct time position.

\section{Self-pulsating PhaseCOMB laser for all-optical clock recovery}

The self-pulsating PhaseCOMB-laser (SP-laser) is a ridge waveguide three section InGaAsP DFB-laser comprising two DFB-sections and an integrated phase tuning section [6]. Each section is driven separately by a direct current. It is a compact device with a total length below $1 \mathrm{~mm}$. The facets of the PhaseCOMB are antireflection coated and the device is packaged into a temperature controlled module.

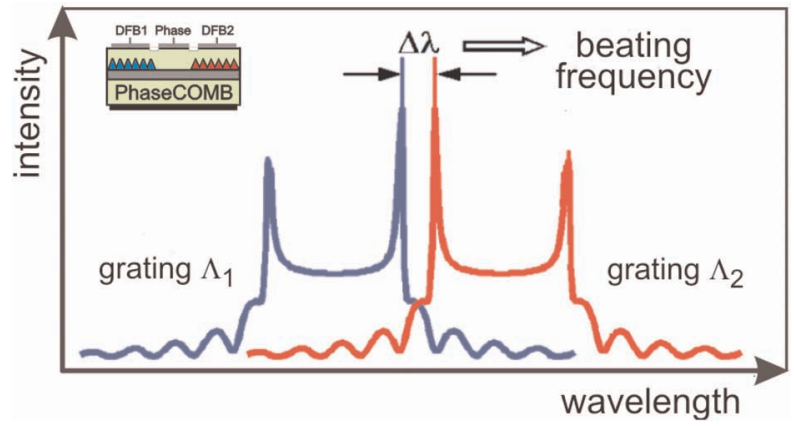

Fig. 9 Design concept of the PhaseCOMB-laser

The superposition of the lasing modes of both DFB-lasers generates a beating signal with a self-pulsation frequency given by their spectral distance (Fig. 9). The frequency can be continuously tuned in a wide range $(28 \mathrm{GHz}-50 \mathrm{GHz})$ simply by the three direct currents [7]. If the frequency is adjusted close to the data rate, the self-pulsation synchronises to the injected PRBS signal

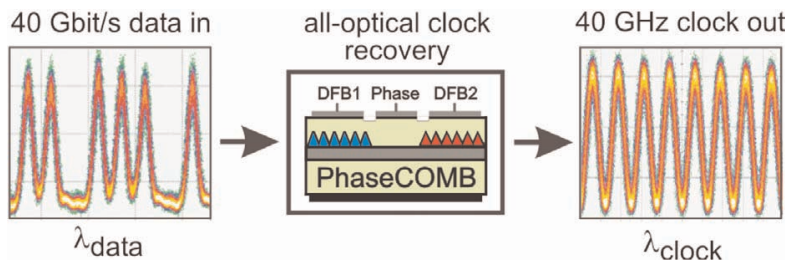

Fig. 10 Operation principle of the self-pulsating laser 


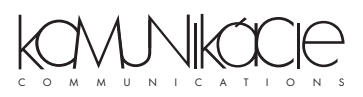

and the SP-laser emits a sinusoidal pulse stream, which is stable in amplitude and time (Fig. 10). The locking to the data signal is polarisation and wavelength insensitive within the whole C-band.

\subsection{Jitter reduction function of the PhaseCOMB laser}

Similar to the previous characterisation of the optically clocked UL-SOA jitter analysis of the self-pulsating laser was performed. The $40 \mathrm{Gbit} / \mathrm{s}$ PRBS data signal at $1550 \mathrm{~nm}$, degraded by additional time jitter, was launched into the PhaseCOMB laser. The SP-laser synchronised to the data signal and recovered a $40 \mathrm{GHz}$ sinusoidal clock signal. The results are summarised in Fig. 11. Note that the time stability of the clock signal is preserved. Even for a data signal with a high jitter of $1400 \mathrm{fs}$, the rms-jitter of the clock remains well below $600 \mathrm{fs}$. Thus the excellent retiming function of the PhaseCOMB-laser is verified.

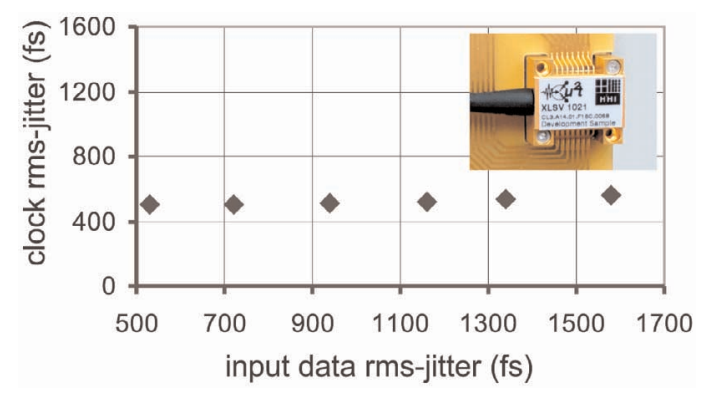

Fig. 11 Time stability of the PhaseCOMB-laser. (The packaged device as insert)

\section{Optically clocked all-optical $3 R$ regenerator}

Finally, the performance of the assembled optically clocked $3 \mathrm{R}$ regenerator (Fig. 2) was investigated. The ideal $40 \mathrm{Gbit} / \mathrm{s} \mathrm{RZ}$ signal $(1550 \mathrm{~nm})$ with a jitter of $520 \mathrm{fs}$ was first additionally degraded by amplitude noise and timing jitter. The strong amplitude fluctuations and the rms-jitter of more than 1200 fs are clearly visible (Fig. 12a). The PhaseCOMB laser locked to the data signal and emitted a stable pulse stream at $1562 \mathrm{~nm}$ with a rmsjitter of only 526 fs (Fig. 12b). According to the optically clocked principle, the degraded data signal and the recovered clock signal were delayed by half a bit relative to each other and launched into the ultra-long SOA. The power levels of the data signal and clock at the UL-SOA input were $-2 \mathrm{dBm}$ and $+4 \mathrm{dBm}$ respectively. The quality of the data signal at the regenerator output is clearly improved (Fig. 12c). The amplitude and time stability of the regenerated output pulses is only marginally affected by the degraded input signal. The power fluctuations are effectively suppressed, the rmsjitter is reduced from 1220 fs to about 650 fs. Furthermore, the regenerated signal has clearly larger eye opening.

Next, bit error rate measurements (BER) were performed to evaluate the system performance of the OC-3R regenerator. The results are shown in Fig. 13. First an ideal back-to-back signal with

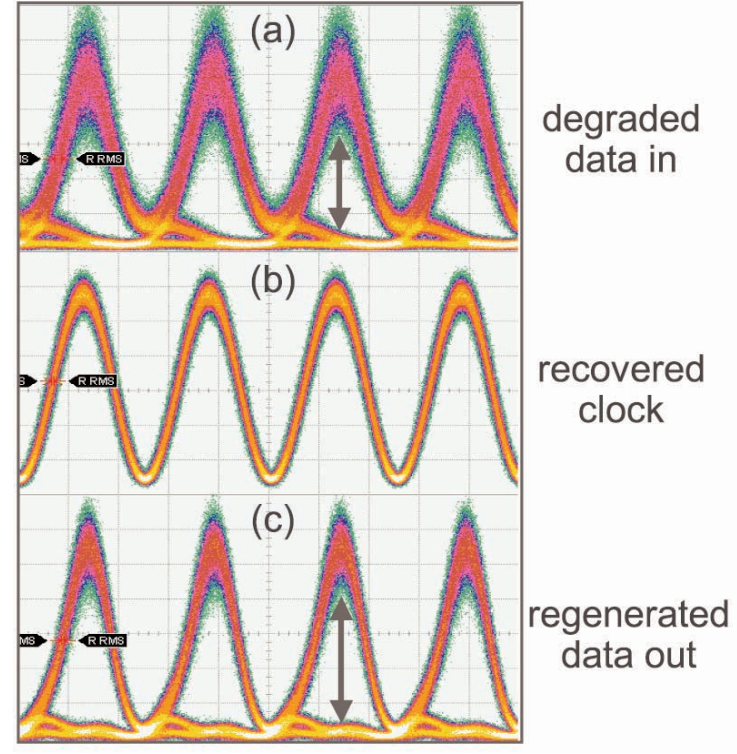

Fig. 12 Degraded $40 \mathrm{Gbit} / \mathrm{s}$ PRBS RZ signal

(a) the recovered $40 \mathrm{GHz}$ clock signal (b) and the data signal at the $O C-3 R$ regenerator output (c)

$520 \mathrm{fs}$ jitter was injected into the bit error rate detector and the BER curve depicted by filled circles was measured. A strongly degraded signal with a jitter of $1220 \mathrm{fs}$ was then applied. The BER curve for the $40 \mathrm{Gbit} / \mathrm{s}$ data signal at the regenerator input is shown by the filled squares and for the output signal by the open squares. The signal quality is clearly improved by the OC-3R regenerator and a negative penalty of more then $-2 \mathrm{~dB}$ is obtained at the bit error rate of 10-9. Note that there is no indication of an error floor down to a BER of 10-11. Further, it can be observed that the BER curve measured for the regenerated signal is close to the BER curve of the ideal input data signal. Thus, the excellent features of the optically clocked $3 R$ regenerator shown by eye analysis were verified by the bit error rate measurements.

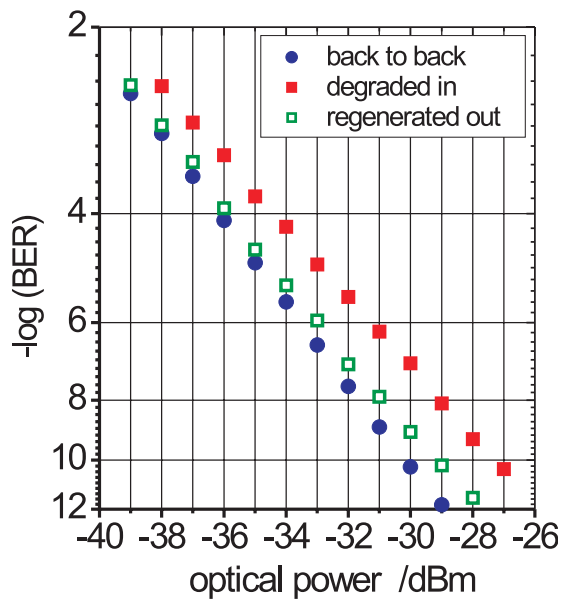

Fig. 13 Performance evaluation of the OC-3R by BER measurements 


\section{Conclusions}

An all-optical 3R regenerator operated in the optically clocked scheme at $40 \mathrm{Gbit} / \mathrm{s}$ has been presented. It was based on two compact semiconductor components: the ultra-long SOA and the self-pulsating PhaseCOMB laser. The dynamic behaviour of the UL-SOA was investigated numerically and experimentally and a good correspondence was found. Applying the optically clocked principle, the carrier density in the SOA was effectively stabilised by the clock signal, resulting in jitter reduction function at the ULSOA output. The self-pulsating DFB-laser applied for all-optical clock recovery synchronised even to strongly degraded data signals and emitted a stable sinusoidal pulse trace. An excellent regenerative function of the assembled optically clocked $3 \mathrm{R}$ regenerator was demonstrated. The rms-jitter of the degraded $40 \mathrm{Gbit} / \mathrm{s}$ data signal was reduced from $1220 \mathrm{fs}$ down to $650 \mathrm{fs}$. The BER curve of the regenerated signal showed a negative penalty of $-2 \mathrm{dBm}$.

The use of semiconductor devices makes the OC-3R regenerator very compact, easy to handle and attractive for application in optical signal processing systems. It will pave the way for competitive and cost effective all-optical 3R schemes.

\section{References}

[1] SARTORIUS, B.: All-Optical 3R Signal Regeneration, ECOC`00, Munich, Germany, We 9.4.1

[2] LAVIGNE. B. ET AL.: Cascade of 100 optical 3R regenerators at 40 Gbit/s based on all-active Mach-Zehnder interferometers, ECOC'01, Amsterdam, Nederland, We.F.2.6

[3] NISHIMURA, K.: Novel All-optical 3R Regenerator Using Cross-Absorption Modulation in RF-Driven Electroabsorption Waveguide, ECOC 2001, Amsterdam, Nederland, We.F.2.4

[4] SLOVAK, J. ET AL.: Optically Clocked Ultra Long SOAs: A Novel Technique for High Speed 3R Signal Regeneration, OFC 2004, Los Angeles, USA, WD4

[5] GIRARDIN, F. ET AL.: Gain recovery of bulk semiconductor optical amplifiers, IEEE Photon. Technol. Lett., vol.10, pp. 784-786, 1998.

[6] SARTORIUS, B.: et al.: All-optical clock recovery module based on self-pulsating DFB laser, Electronics Lett., 20th August 1998, Vol. 36, No. 17

[7] SARTORIUS, B.: All-Optical Clock Recovery for 3R Optical Regeneration, OFC 2001, Anaheim, California, MG 7, invited. 